\title{
Anaesthetic management of a premature low-birth- weight neonate with congenital complete heart block for implantation of temporary epicardial pacing wires
}

Oriana $\mathrm{Ng}^{1}$, MBBS, MMed, Shahani Jagdish Menghraj², MBBS, MD

\begin{abstract}
The optimal anaesthetic management of neonates with complete congenital heart block (CCHB) is unknown, as there is a low incidence of such cases. Neonates with CCHB often require surgery for the initiation of electronic pacing. In addition to the challenges of anaesthetising a neonate, this procedure is risky due to the potential for hypotension, arrhythmias and cardiac arrest. We herein present the case of a premature low-birth-weight neonate with antibody-related $\mathrm{CCHB}$ and normal heart structure who underwent anaesthesia and surgery for epicardial pacing wire insertion on Day 1 of life. We also compare our patient's anaesthetic conduct and outcome with similar previously reported cases.
\end{abstract}

Keywords: anaesthesia, complete congenital heart block, epicardial pacing wire, neonate, premature

\section{INTRODUCTION}

Complete congenital heart block (CCHB) is a rare condition with an incidence of 1 in 20,000 live births. ${ }^{(1)}$ Neonates with $\mathrm{CCHB}$ often require surgery (and therefore anaesthesia) for the insertion of temporary or permanent electronic pacing wires. As this condition occurs infrequently, the most favourable method of induction and maintenance of anaesthesia is unclear, although several techniques have been used. We herein report the anaesthetic conduct of a low-birth-weight neonate who underwent temporary epicardial pacing wire insertion with good outcome.

\section{CASE REPORT}

The mother of our patient was a 24-year-old Malay woman with no prior known medical problem. She had previously undergone a full term pregnancy that culminated in a normal vaginal delivery of a healthy baby three years prior to the current presentation. During this current pregnancy, screening at 23 weeks of gestation demonstrated fetal heart block with an atrial rate of 130-140 bpm and a ventricular rate of 48-51 bpm. The mother was subsequently found to be positive for antiribonucleoprotein, anti-Ro, anti-La and antinuclear antibodies, but negative for anti-ds DNA, anti-Smith, anti-Scl-70 and anti-Jo-1 antibodies. She was diagnosed with Sjögren's syndrome, and was started on $8 \mathrm{mg}$ dexamethasone once every morning and $4 \mathrm{mg}$ salbutamol three times a day at 25 weeks of gestation in an attempt to increase the fetal heart rate. However, salbutamol was stopped a week later as the mother experienced cardiovascular side effects and there was no change in the fetal ventricular rate. Subsequent follow-up imaging demonstrated fetal cardiomegaly and pericardial effusion ranging from $2.9 \mathrm{~cm}$ to $5.0 \mathrm{~cm}$ in thickness, but no evidence of fetal hydrops. In view of oligohydramnios, increasingly high uterine artery pressure indices and evidence of intrauterine growth restriction, a decision was made to deliver the neonate via Caesarean section at 34 weeks of gestation.

Our patient was female and born with a weight of 1,545 g. She had Apgar scores of 7 and 8 at 1 and 5 mins of life, respectively. Her heart rate was $60 \mathrm{bpm}$ at birth, slowing to $40 \mathrm{bpm}$ by the third minute of life. Oxygen saturation was adequate at room air. Umbilical arterial and venous lines were inserted and an isoprenaline infusion of up to $0.3 \mu \mathrm{g} / \mathrm{kg} / \mathrm{min}$ was started. The neonate's heart rate reached a peak of $60 \mathrm{bpm}$ on this therapy. However, the dose of the isoprenaline infusion could not be increased beyond $0.3 \mu \mathrm{g} / \mathrm{kg} / \mathrm{min}$, as the patient developed hypotension at higher doses. There was no other manifestation of neonatal lupus and blood investigations were within normal limits. Chest radiography showed slight cardiomegaly but clear lung fields. Transthoracic echocardiography showed a structurally normal heart with a fractional shortening of $34 \%$, a tiny pericardial effusion (measuring $2.1 \mathrm{~mm}$ ) and a large patent ductus arteriosus with bidirectional flow.

As our patient was haemodynamically stable, she was listed for epicardial ventricular pacing in the elective theatre the following morning and nursed overnight in the neonatal intensive care unit (NICU) with continuous pulse oximetry, three-lead electrocardiography and umbilical arterial pressure monitoring. Our patient was not premedicated. In addition to the umbilical catheters, she arrived at the operating theatre with a 24G intravenous cannula in her left hand. Monitoring

${ }^{1}$ Department of Anaesthesiology, Singapore General Hospital, ${ }^{2}$ Paediatric Anaesthesia, KK Women's and Children's Hospital, Singapore

Correspondence: Dr Oriana Ng, Associate Consultant, Department of Anaesthesiology, Block 6, Level 2, Singapore General Hospital, Outram Road, Singapore 169608. oriana.ng@sgh.com.sg 
(i.e. pulse oximetry, electrocardiography, and umbilical arterial monitoring) was continued from NICU, throughout the operation. Isoprenaline at $0.3 \mu \mathrm{g} / \mathrm{kg} / \mathrm{min}$ was continued. After preoxygenation, $10 \mathrm{mg}$ of thiopentone was administered, followed by $1 \mathrm{mg}$ of atracurium after confirming the ability to manually ventilate the patient. The patient was intubated orally with a 3.0 uncuffed endotracheal tube secured at $8 \mathrm{~cm}$ at the gums. Transcutaneous defibrillation and pacing pads were placed over the midscapular region and the right lateral hemithorax. The small size of the patient necessitated trimming of the periphery of the defibrillation pads (done without compromising the contact gel surface area) in order to make room for surgery and other monitors. The diathermy pad was placed around the right thigh of the neonate, and the surgery was carried out in the supine position. Anaesthesia was maintained with oxygen on air at the following levels: fraction of inspired oxygen at $55 \%$, sevoflurane up to $1.5 \%$, and fentanyl up to $5 \mu \mathrm{g} / \mathrm{kg}$. Limited lower sternotomy was performed and two epicardial pacing wires were placed over the right ventricle. Bipolar pacing was set on VOO mode, at a threshold of $7 \mathrm{~mA}$ and a rate of $130 \mathrm{bpm}$. After establishing capture, the isoprenaline infusion was ceased. The durations of the anaesthesia and surgery were 100 and 50 mins, respectively. The entire procedure was uneventful. No reversal agents were administered. The patient was kept intubated and sedated on morphine infusion upon return to NICU and extubated 15 hours after the procedure. By Day 20 of life, the threshold of the pacemaker had increased to $12 \mathrm{~mA}$, but otherwise it was functioning well. The patient currently awaits implantation of a long-term pacemaker once a body weight of $3 \mathrm{~kg}$ is achieved.

\section{DISCUSSION}

The incidence of CCHB is estimated at 1 in 20,000 live births, of which $30 \%$ of cases have an associated structural defect. $^{(1)}$ Isolated $\mathrm{CCHB}$ is often associated with maternal connective tissue diseases, which was the case in our patient. Neonatal lupus syndrome occurs when the mother's anti-Ro or anti-La antibodies accumulate in the fetal circulation, resulting in fibrotic replacement of the conducting tissue. In such situations, $\mathrm{CCHB}$ can be associated with neonatal lupus rash, cytopenia or liver enzyme imbalances. However, these aforementioned associations were absent in our patient. There can be associated myelopathy and pulmonary involvement, although rare.(2) Other rarer causes of $\mathrm{CCHB}$ include fetal myocarditis, mitochondrial disease, 18p syndrome and idiopathic heart block. ${ }^{(1)}$ It is important that the underlying cause of the $\mathrm{CCHB}$ is sought during preoperative assessment.

Mortality before 3 months of age is at $19 \%$ and more than $65 \%$ of surviving neonates require pacemakers. Morbidity in $\mathrm{CCHB}$ is primarily a result of the development of fetal hydrops, which requires drainage of effusions at birth, as well as immediate pharmacological or electrical pacing. Maternal steroids are often used to improve effusions, ascites and hydrops, but this is controversial as it is not completely efficacious and may result in fetal complications such as intrauterine growth retardation, oligohydramnios, prolonged adrenal suppression, and late learning disabilities. ${ }^{(1)}$

The risks of $\mathrm{CCHB}$ include bradycardia-induced hypotension and cardiac arrest, especially during anaesthesia. Invasive cardiovascular monitoring is therefore patently indicated. Intraoperative hypotension can occur in structurally normal hearts despite inotropic support, as cardiac output depends on heart rate. Drugs often used to counter bradycardia in such conditions include isoprenaline, adrenaline, dopamine and atropine. However, the response to these medications is usually insignificant, as evidenced in our patient..$^{(3)}$ In view of this, temporary pacing via the transcutaneous, epicardial or transvenous route is often instituted until the infant is of sufficient size for the permanent insertion of an epicardial pacing system. The decision to initiate temporary pacing in the present case was the low birth weight of the child, which precluded insertion of a permanent pacemaker. In the largest published series of $\mathrm{CCHB}$ cases, the lowest weight at which a permanent pacemaker has been inserted is $1.9 \mathrm{~kg}$, while temporary pacing has been inserted in a neonate as small as $1.0 \mathrm{~kg} .{ }^{(1)}$

In our patient, a limited lower sternotomy was made. Other possible surgical approaches include full sternotomy, subxiphoid incision, left anterior thoracotomy, and left posterolateral thoracotomy; the latter two are more commonly used when further cardiovascular procedures are being carried out in the same setting. ${ }^{(1)}$ The anaesthetist should ascertain the best location for surgical incision because of its impact on where the electrocardiographic leads and transcutaneous pacing pads can be placed.

Many types of anaesthesia have been used for pacing wire insertion, including narcotic anaesthesia with fentanyl supplemented with nitrous oxide, midazolam, intravenous ketamine, thiopentone, isoflurane and sevoflurane. However, none of these types of anaesthesia have been demonstrated to be superior over another. ${ }^{(1)}$ In our case, thiopentone was chosen as an induction agent over a volatile anaesthetic, as thiopentone produces less myocardial depression and cardiac dysrhythmia is unlikely with the use of thiopentone. At our institution, ketamine is usually avoided in infants due to concerns about the drug's effect on neurodevelopment. The child was not premedicated with atropine as this is not routine practice at our institution. Furthermore, the use of atropine in such clinical scenarios has been known to result in a less than $20 \%$ increase in heart rate. ${ }^{(1)}$ This may be due to the fact that while the associated vagolysis may increase atrial rate, atrioventricular conduction is unlikely to be improved in established complete heart block.

Castilla et al reported the use of a size 1 laryngeal mask airway (LMA) in a 33-week-old baby weighing $2.05 \mathrm{~kg} .^{(4)}$ Their rationale behind this was the need to maintain baseline cardiorespiratory stability of the patient, as well as to maintain spontaneous respiration, thus obviating the need for paralysis for intubation or exposure to reversal agents. ${ }^{(4)}$ Although this is 
an attractive idea, we did not adopt the same technique for our patient as we were concerned about the optimal positioning of the LMA and the relatively high potential for dislodgement or obstruction in a 1.5-kg neonate under surgical drapes. Furthermore, we did not expect the duration of postoperative intubation to be inordinately prolonged, and pharmacological manipulation of the heart rate would be a less significant issue once electrical pacing was initiated.

Previous reports on intraoperative management did not discuss management of possible arrhythmia or cardiac arrest before the successful installation of pacing wires. In our case, we elected to apply transcutaneous defibrillation/pacing pads on our patient before the start of surgery. We found that the pads had to be trimmed to an appropriate size (while maintaining the area and integrity of the contact gel) for our patient. Without our decision to apply transcutaneous defibrillation/pacing pads on our patient, we may not have considered in advance the important problem of the pads being too large for easy and rapid placement on a low-birth-weight neonate in a crisis. Indeed, although none of the cases described underwent transcutaneous pacing, the provision of facilities for this purpose before induction was recommended in the largest series of CCHB cases in the literature. ${ }^{(1,5)}$ The use of prophylactic temporary transvenous pacing has been reported in a $3.6-\mathrm{kg}$ neonate with $\mathrm{CCHB}$ who underwent exploratory laparotomy. ${ }^{(6)}$ This method was chosen by Sreevastava et al due to the nonavailability of a transcutaneous pacing system for an infant of this size. ${ }^{(6)}$ Although we posit that the transcutaneous method is potentially feasible in such situations and is preferable to the more invasive transvenous method, we were fortunate enough not to have to put our modified pads to the test. Thus, in light of this, the feasibility of transcutaneous pacing in the neonatal population would be a possible area for future investigation.

Our patient remained haemodynamically stable throughout the procedure. While pharmacological considerations had been made to achieve this, it is also likely that the absence of structural cardiac lesions contributed to the patient's overall stability.

\section{REFERENCES}

1. Kussman BD, Madril DR, Thiagarajan RR, Walsh EP, Laussen PC. Anesthetic management of the neonate with congenital complete heart block: a 16year review. Pediatr Anaesth 2005; 15:1059-66.

2. Friedman DM, Rupel A, Buyon JP. Epidemiology, etiology, detection, and treatment of autoantibody-associated congenital heart block in neonatal lupus. Curr Rheumatol Rep 2007; 9:101-8.

3. Deloof E, Devlieger H, Van Hoestenberghe R, et al. Management with a staged approach of the premature hydropic fetus due to complete congenital heart block. Eur J Pediatr 1997; 156:521-3.

4. Castilla M, Jerez M, Llacer M, Martinez S. Anaesthetic management in a neonate with congenital complete heart block. Paediatr Anaesth 2004; $14: 172-5$.

5. Das SN, Kale SC. Perioperative management of pacemaker implantation for congenital complete heart block. J Cardiothorac Vasc Anesth 2004; 18:628-9.

6. Sreevastava DK, Setlur R, Sharma VK, et al. Anesthetic management of an infant with lupus and congenital complete heart block. Paediatr Anaesth 2006; 16:216-7. 